Research Article

\title{
Investigation of the Interaction of Herbal Ingredients Contained in Triphala Recipe Using Simplex Lattice Design: Chemical Analysis Point of View
}

\author{
Chaowalit Monton $\mathbb{D}^{1},{ }^{1}$ Thaniya Wunnakup, ${ }^{1}$ Jirapornchai Suksaeree, ${ }^{2}$ \\ Laksana Charoenchai, ${ }^{1}$ and Natawat Chankana ${ }^{3}$ \\ ${ }^{1}$ Drug and Herbal Product Research and Development Center, College of Pharmacy, Rangsit University, \\ Pathum Thani 12000, Thailand \\ ${ }^{2}$ Department of Pharmaceutical Chemistry, College of Pharmacy, Rangsit University, Pathum Thani 12000, Thailand \\ ${ }^{3}$ Sun Herb Thai Chinese Manufacturing, College of Pharmacy, Rangsit University, Pathum Thani 12000, Thailand
}

Correspondence should be addressed to Chaowalit Monton; chaowalit@rsu.ac.th

Received 16 March 2020; Revised 19 June 2020; Accepted 16 July 2020; Published 4 August 2020

Academic Editor: Carl J. Schaschke

Copyright (c) 2020 Chaowalit Monton et al. This is an open access article distributed under the Creative Commons Attribution License, which permits unrestricted use, distribution, and reproduction in any medium, provided the original work is properly cited.

\begin{abstract}
The aim of this work was to investigate the interaction of herbal ingredients contained in Triphala recipe (Terminalia chebula, Terminalia bellirica, and Phyllanthus emblica in equal proportion) using simplex lattice design. This work focused on chemical analysis of four phenolic compounds including gallic acid, corilagin, chebulagic acid, and chebulinic acid by validated highperformance liquid chromatography. The effect of the extraction technique (decoction vs. infusion) and gamma irradiation was also examined. The combination index was used as a tool for determination of interaction of the ingredients contained in the herbal recipe. Results showed that the extraction technique and gamma irradiation slightly altered the content of some phenolic compounds as well as the combination index. The positive interaction seems to be found at the equal proportion of the three plants. This work scientifically supported the suitable formula of the Triphala recipe in the traditional use.
\end{abstract}

\section{Introduction}

Triphala is an herbal formula composed of equal proportion of dried fruits of three plants: Terminalia chebula Retz. var. chebula, Terminalia bellirica (Gaertn.) Roxb., and Phyllanthus emblica L. It has been used for a long time in Ayurvedic medicines. Triphala possesses numerous activities, including adaptogenic, antibacterial, antidiabetic, anti-inflammation, antimutagenic, antineoplastic, antioxidant, appetite stimulation, chemoprotective, immunomodulating, laxative, prevention of dental caries, radioprotective, and reduction of hyperacidity [1]. Triphala contains high content of ascorbic acid and phenolic compounds, so it possesses good antioxidant activity [2]. Pawar et al. [3] reported the use of gallic acid, chebulagic acid, and chebulinic acid as standard markers for Triphala. Charoenchai et al. [4] report the high-performance liquid chromatography (HPLC)- mass spectrometry profiles of Triphala and modified Triphala recipes. The major active compounds of Triphala are ascorbic acid, gallic acid, corilagin, chebulagic acid, rutin, chebulinic acid, and quercetin. Varma et al. [5] also reported the chemical constituents, including gallic acid, ellagic acid, chebulinic acid, emblicanin A and B, and friedelin. The other phenolic compounds were also reported, i.e., chebulic acid, tannic acid, epicatechin, and syringic acid [2]. Phenolic compounds usually act as an antioxidant; the high content of phenolic compounds has a positive effect on antioxidant activity [6-9]. Several studies reveal that Triphala is an antioxidant-rich product so that it can be used as a food supplement and beverage to promote health [10].

Synergistic effects or positive interaction of drugs or herbal plants enhance therapeutic effects or biological activities by some positive interactions among their different components [11]. The synergism is reported among herbal plants in numerous studies [12-19]. In some reports, herbal extract, as 
<smiles>O=C(O)c1cc(O)c(O)c(O)c1</smiles>

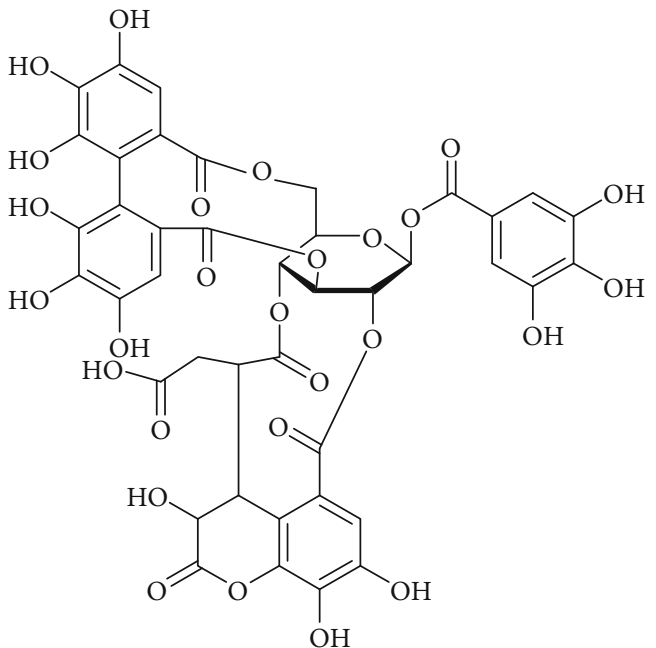

Chebulagic acid

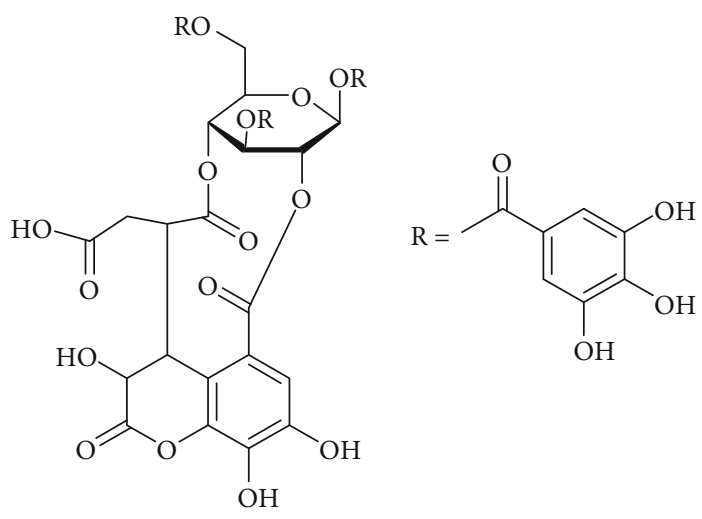

Chebulinic acid

Figure 1: Chemical structures of phenolic compounds (gallic acid, corilagin, chebulagic acid, and chebulinic acid).

well as herbal plant, is synergized with some modern medicines, especially, essential oils with antibiotic [20-22]. Among several traditional medicines, traditional Chinese medicines are the most frequently reported in the literature to exhibit synergism of herbal plants [23, 24]. A synergistic effect in traditional Chinese medicines is reviewed. Multicomponent combinations of herbal plants are usually used in traditional medicines to boost the therapeutic efficacy and reduce side effects. The synergism can occur among herbs or other components in the prescription, among effective parts of herbs, and among bioactive compounds of herbs [25].

Triphala was previously reported for synergism with gentamicin or oxacillin against multidrug resistant gramnegative bacilli or multidrug resistant Staphylococcus aureus, respectively [26]. However, there is no report accounting for the synergism among the plant ingredients containing in its formula. So the aim of this work was to investigate the interaction of herbal ingredients contained in the Triphala recipe using simplex lattice experimental design. The 3D response surface analysis was applied to provide a complete description of the combination effect [27]. This work focused on the chemical analysis of four phenolic compounds, including gallic acid, corilagin, chebulagic acid, and chebulinic acid by using validated HPLC. Chemical structures of these phenolic compounds are shown in Figure 1. The effects of the extrac- tion technique (decoction vs. infusion) and gamma irradiation (irradiation vs. nonirradiation) were also studied. The combination index was used as a tool for the determination of the interaction of the ingredients contained in the Triphala recipe. The authors expected that the data obtained from this work could be used to support the ratio of herbal ingredients contained in the Triphala recipe in traditional use.

\section{Materials and Methods}

2.1. Materials. The four reference standards, i.e., gallic acid (purity 99.88\%), corilagin (purity 99.67\%), chebulagic acid (purity 99.62\%), and chebulinic acid (purity 98.42\%), were purchased from Chengdu Biopurify Phytochemicals Ltd., China. The solvents were analytical and HPLC grades.

2.2. Preparation of Plant Samples. Dried fruits of T. chebula, T. bellirica, and P. emblica were purchased from Charoensuk Osod, Nakhon Pathom Province, Thailand. They were authenticated by Ajarn Nirun Vipunngeun, plant taxonomist and lecturer at Department of Pharmacognosy, College of Pharmacy, Rangsit University. T. chebula, T. bellirica, and P. emblica were deposited at the Drug and Herbal Product Research and Development Center, College of Pharmacy, Rangsit University. The voucher specimens were coded as CM-TC001-1-04-2019, 
CM-TB001-1-04-2019, and CM-PE001-1-04-2019, respectively. Seeds were removed from the fruits of each plant. The obtained fruits were pulverized using a grinder and stored in a dry place until use.

\subsection{Simplex Lattice Experimental Design for Extraction of} Plant Samples and the Optimization. The simplex lattice design was applied in this work. The weight fraction of $T$. chebula, T. bellirica, and P. emblica ranged from 0 to 1 , and the summation of the weight fraction of the three plants was equal to 1 . The independent variables of this work were the proportion of T. chebula $\left(X_{1}\right), T$. bellirica $\left(X_{2}\right)$, and $P$. emblica $\left(X_{3}\right)$. The 12 formulas of plant mixture were prepared: F1 to F9 contained T. chebula, T. bellirica, and $P$. emblica in proportions of $1: 0: 0,0: 1: 0,0: 0: 1,0.5: 0.5: 0$, $0.5: 0: 0.5,0: 0.5: 0.5,0.66: 0.17: 0.17,0.17: 0.66: 0.17$, and $0.17: 0.17: 0.66$, respectively. F10 to F12 were the same formula. They contained equal proportion of $T$. chebula, $T$. bellirica, and P. emblica $(0.33: 0.33: 0.33)$.

The plant powder mixture was used for extraction by decoction and infusion methods. In case of decoction, plant powder mixture $(6 \mathrm{~g})$ was placed in a tea bag and boiled in water $(50 \mathrm{~mL})$ for $15 \mathrm{~min}$. The tea bag with powder mixture was removed and boiled again three times in total. The three parts of the obtained solution were pooled and filtered through Whatman ${ }^{\circledR}$ filter paper no.1. It was lyophilized using a freeze dryer for 18-24 hours. The lyophilized extract powder was collected and kept in desiccator until use.

In case of infusion, plant powder mixture $(6 \mathrm{~g})$ was placed in a tea bag, placed in boiling water $(50 \mathrm{~mL})$, and stood for $15 \mathrm{~min}$. The tea bag with powder mixture was removed and infused again three times in total. The three parts of the obtained solution were pooled and filtered. It was lyophilized similar to the decoction group. The lyophilized extract powder was collected and kept in desiccator until use.

The extract powder obtained from decoction was sampled to gamma irradiation ( $10 \mathrm{kGy})$. The three groups of samples, i.e., extract from decoction without gamma irradiation, extract from decoction with gamma irradiation, and extract from infusion, were obtained. They have analyzed for content of phenolic compounds, including gallic acid, corilagin, chebulagic acid, and chebulinic acid. The six dependent variables, i.e., extraction yield $\left(Y_{1}\right)$, gallic acid content $\left(Y_{2}\right)$, corilagin content $\left(Y_{3}\right)$, chebulagic acid content $\left(Y_{4}\right)$, chebulinic acid content $\left(Y_{5}\right)$, and total content of the four phenolic compounds $\left(Y_{6}\right)$ were monitored. They were used to produce the 3D response surface by Design-Expert ${ }^{\circledR}$ version 11.0. Furthermore, the equations for prediction of each dependent factor were created.

2.4. Analysis of Phenolic Compounds. The HPLC was used to analyze the four phenolic compounds, including gallic acid, corilagin, chebulagic acid, and chebulinic acid. The aqueous solutions of the extract of F1 to F12 were prepared in a concentration of $2 \mathrm{mg} / \mathrm{mL}$, except F3 which was prepared in a concentration of $0.5 \mathrm{mg} / \mathrm{mL}$, and F6 and F9 were prepared in a concentration of $1 \mathrm{mg} / \mathrm{mL}$. The content of the four phenolic compounds was calculated based on the calibration curve of each compound.
The analysis of phenolic compounds was performed using an HPLC instrument (Agilent 1260 infinity, Agilent, USA). The ACE C18-PFP column $(250 \times 4.6 \mathrm{~mm}$, internal diameter, $5 \mu \mathrm{m}$ ) was used. The column temperature was controlled at $25^{\circ} \mathrm{C}$. The mobile phase was composed of acetonitrile (A) and 1\% acetic acid aqueous solution (B). The gradient elution system was similar to the previous work of Charoenchai et al. [4]. It was started by holding 5\% A for $1 \mathrm{~min}$, increased to $10 \% \mathrm{~A}$ in $3 \mathrm{~min}$, increased to $15 \% \mathrm{~A}$ in $8 \mathrm{~min}$, increased to $35 \% \mathrm{~A}$ in $20 \mathrm{~min}$, increased to $50 \% \mathrm{~A}$ in $3 \mathrm{~min}$, increased to $100 \% \mathrm{~A}$ in $2 \mathrm{~min}$ and holding for $3 \mathrm{~min}$, and decreased to $5 \% \mathrm{~A}$ in $1 \mathrm{~min}$ and holding for $4 \mathrm{~min}$. The mobile phase flow rate was $1 \mathrm{~mL} / \mathrm{min}$. The injection volume was $10 \mu \mathrm{L}$. The photodiode array detector was set at $270 \mathrm{~nm}$.

This method was validated to confirm its linearity, range, the limit of detection (LOD) and limit of quantitation (LOQ), specificity, precision, and accuracy. The linear equations and coefficient of determination $\left(R^{2}\right)$ values of calibration curves of gallic acid, corilagin, chebulagic acid, and chebulinic acid in the test range of $10-200 \mu \mathrm{g} / \mathrm{mL}$ were $y=$ $4039955 x+6926719\left(R^{2}=0.9991\right), y=2292681 x+9273157$ $\left(R^{2}=0.9978\right), y=1672107 x+197458\left(R^{2}=1.0000\right)$, and $y=$ $2068580 x-298060\left(R^{2}=0.9999\right)$, respectively. The LOD and LOQ values were 2.50 and $7.57 \mu \mathrm{g} / \mathrm{mL}, 0.46$ and $1.39 \mu \mathrm{g} / \mathrm{mL}, 0.98$ and $2.96 \mu \mathrm{g} / \mathrm{mL}$, and 0.84 and $2.55 \mu \mathrm{g} / \mathrm{mL}$, respectively. The percent relative standard deviations of intraday precision and interday precision of all phenolic compound standards were lower than $2 \%$ and $5 \%$, respectively. The accuracy values of the analysis of gallic acid, corilagin, chebulagic acid, and chebulinic acid were 95.23-105.2\%, 91.74-107.0\%, 99.22-104.3\%, and 92.98-101.2\%, respectively. Furthermore, this method was specifically due to the UV spectrums of the individual peak of each phenolic compound in the extract similar to the UV spectrum of each standard compounds.

2.5. Investigation of Interaction. The interaction was investigated based on the response additivity approach, which is also referred to as the linear interaction effect. The posotove interaction occurred when the observed combination effect was higher than the expected additive effect given by the summation of the individual effect [27]. However, this work focused on the content of phenolic compounds, so the positive interaction in this work occurred when the observed content of phenolic compounds from the combination plants was higher than the expected additive content given by the summation of the individual plant. The tool used to investigate the interaction was adapted from the combination index (CI), a practical model used for determination of synergism of a multicomponent mixture in a fixed ratio [24], as

$$
\mathrm{CI}=\frac{E_{A}+E_{B}+E_{C}}{E_{\text {combination }}},
$$

where $E_{A}, E_{B}, E_{C}$ were the individual effect of T. chebula, T. bellirica, and $P$. emblica, respectively. $E_{\text {combination was the }}$ observed combination effect of T. chebula, T. bellirica, and P. emblica. The positive interaction, additive effect, and negative interaction occurred when the CI values were lower than 1 , equal to 1 , and higher than 1 , respectively $[24,27$. 


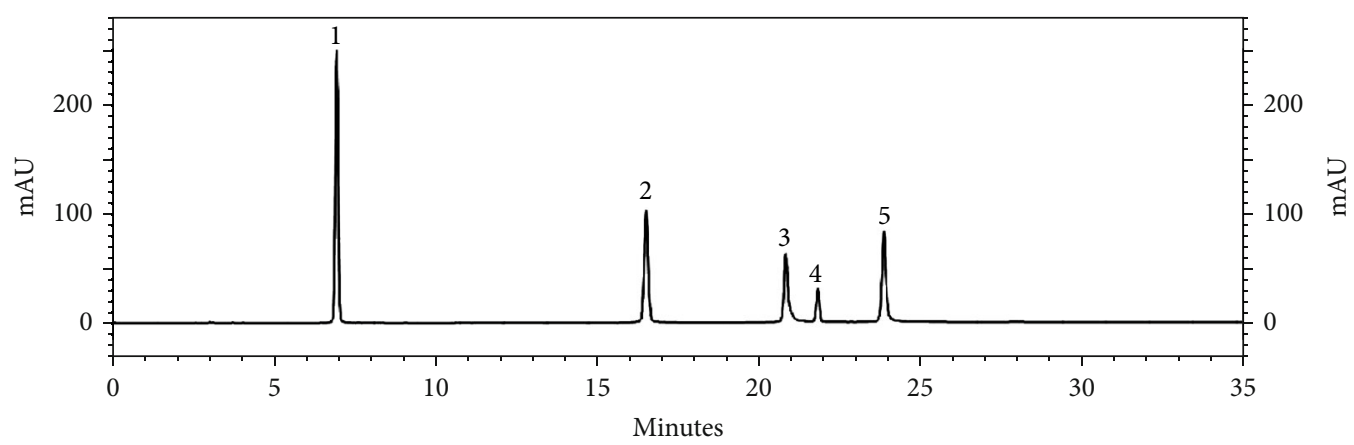

(a)

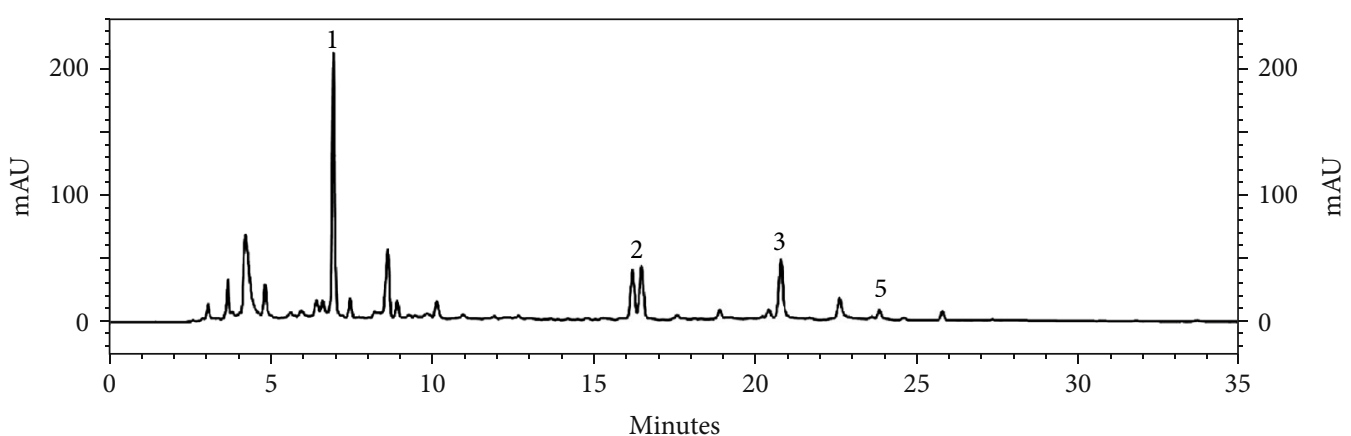

(b)

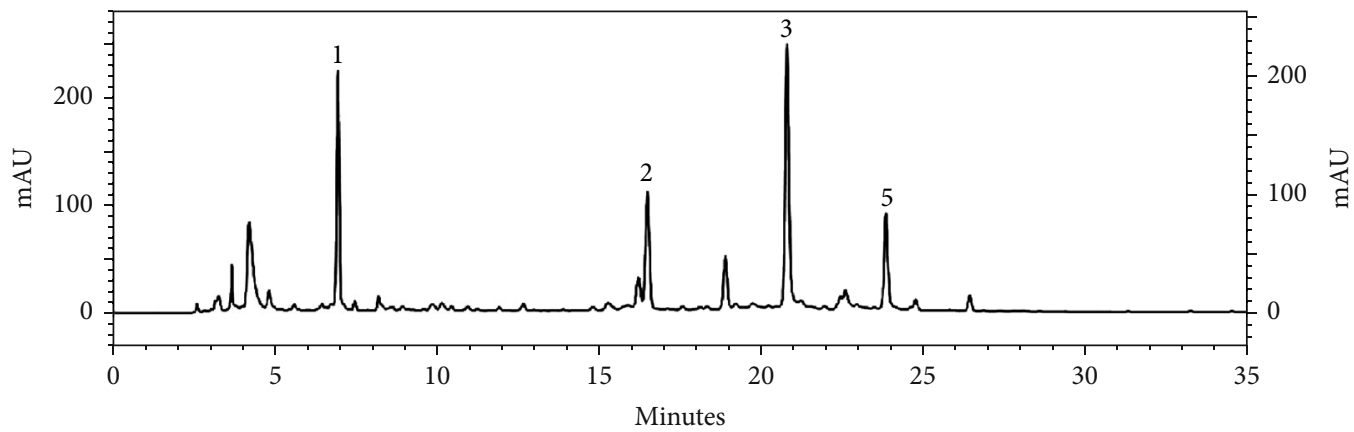

(c)

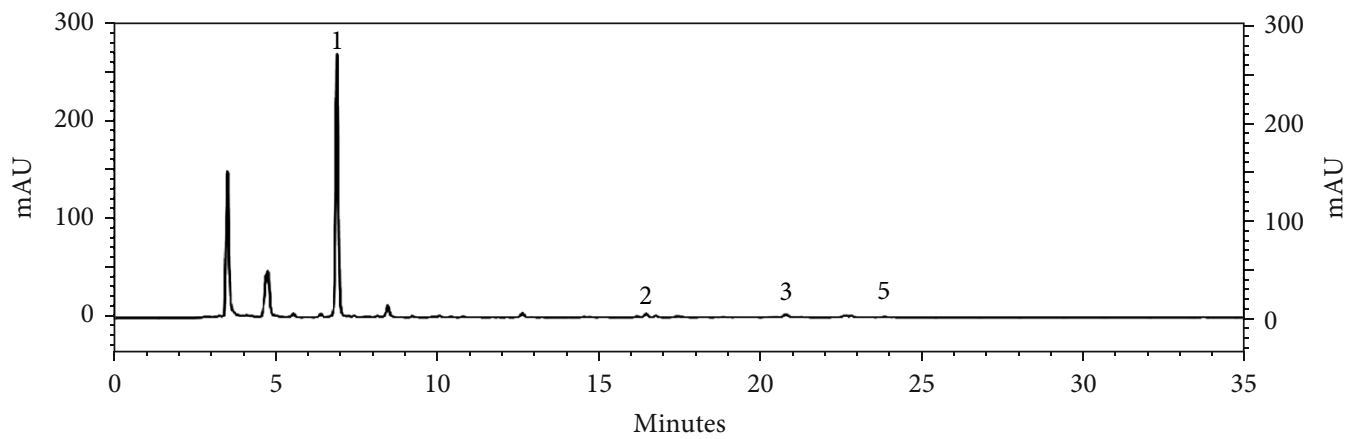

(d)

Figure 2: Continued. 


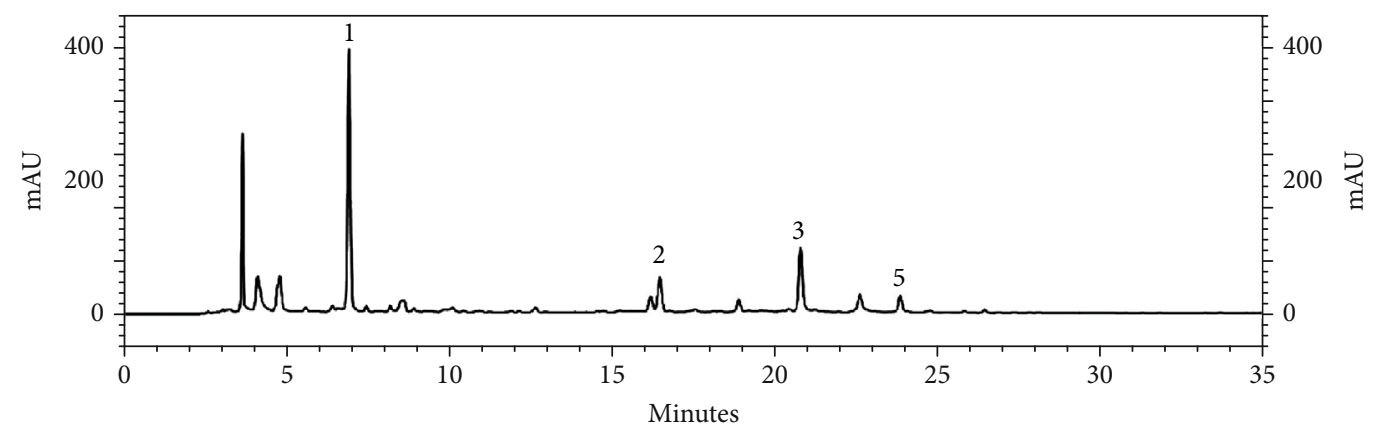

(e)

FIgURe 2: HPLC chromatograms of (a) mixed standards of gallic acid (1), corilagin (2), chebulagic acid (3), rutin (4), and chebulinic acid (5) in each concentration of $50 \mu \mathrm{g} / \mathrm{mL}$, (b) F1 decoction extract $(2 \mathrm{mg} / \mathrm{mL}$ ), (c) F2 decoction extract $(2 \mathrm{mg} / \mathrm{mL})$, (d) F3 decoction extract $(0.5 \mathrm{mg} / \mathrm{mL})$, and (e) F10 decoction extract $(2 \mathrm{mg} / \mathrm{mL})$.

The response surface methodology was also applied to clarify the interaction. The six dependent variables of CI values of the extraction yield $\left(Y_{7}\right)$, gallic acid content $\left(Y_{8}\right)$, corilagin content $\left(Y_{9}\right)$, chebulagic acid content $\left(Y_{10}\right)$, chebulinic acid content $\left(Y_{11}\right)$, and total content of the four phenolic compounds $\left(Y_{12}\right)$ were monitored. They were used to produce the contour plots by Design-Expert ${ }^{\circledR}$ version 11.0. The equations for the prediction of each dependent variable were created. The plots between the predicted values and the actual values were produced, and the $R^{2}$ values were reported to explain the level of correlation. The plots between internally studentized residuals and the run numbers were also produced to demonstrate the level of the distribution of the data. The contour plots of the desirability of the optimal condition provided the simultaneous minimizing CI of the extraction yield $\left(Y_{7}\right)$, and total content of the four phenolic compounds $\left(Y_{12}\right)$ was created. Finally, the overlay plots that CI values of both extraction yield and total content of four phenolic compounds of less than 0.5 and 0.4 were reported.

\section{Results and Discussion}

3.1. Content of the Phenolic Compounds. Charoenchai et al. [4] reported that chemical constituents contained in Triphala obtained from electrospray mass spectrometry were ascorbic acid, gallic acid, corilagin, chebulagic acid, rutin, chebulinic acid, and quercetin (small amount). The five phenolic compounds, including gallic acid, corilagin, chebulagic acid, rutin, and chebulinic acid were selected as standard markers for Triphala used in this study. The previous work demonstrated that HPLC could be used as an important instrument for quantitation of chemical constituents in the Triphala recipe. The HPLC chromatograms of mixed standards and the formulas are shown in Figure 2. The highest chemical compound found in T. chebula and P. emblica was gallic acid, while the highest chemical compound of $T$. bellirica was chebulagic acid. However, rutin was not found in all 12 formulas. So only gallic acid, corilagin, chebulagic acid, and chebulinic acid were further quantified.

The mathematic equations used to predict each response $\left(Y_{1}\right.$ to $\left.Y_{6}\right)$ are shown in Equations (2)-(19). Among the proportion of T. chebula $\left(X_{1}\right), T$. bellirica $\left(X_{2}\right)$, and $P$. emblica $\left(X_{3}\right)$, the $X_{2}$ had the highest effect on the extraction yield $\left(Y_{1}\right)$, corilagin content $\left(Y_{3}\right)$, chebulagic acid content $\left(Y_{4}\right)$, chebulinic acid content $\left(Y_{5}\right)$, and total content of the four phenolic compounds $\left(Y_{6}\right)$ of all groups, except, $Y_{1}$ of the infusion group was affected the greatest by the $X_{1}$. According to the gallic acid content $\left(Y_{2}\right)$ of all groups, they were the greatest affected by $X_{3}$.

\subsubsection{Decoction without Gamma Irradiation.}

$$
\begin{aligned}
Y_{1}= & 17.56 X_{1}+20.89 X_{2}+16.38 X_{3}+90.24 X_{1} X_{2} \\
+ & 67.88 X_{1} X_{3}+67.88 X_{2} X_{3}+114.00 X_{1} X_{2} X_{3}, \\
Y_{2}= & 0.29 X_{1}+0.40 X_{2}+1.47 X_{3}+2.32 X_{1} X_{2} \\
& +2.81 X_{1} X_{3}+4.97 X_{2} X_{3}, \\
Y_{3}= & 0.10 X_{1}+0.49 X_{2}+0.08 X_{3}+1.48 X_{1} X_{2} \\
& +0.21 X_{1} X_{3}+2.05 X_{2} X_{3}-3.81 X_{1} X_{2} X_{3}, \\
Y_{4}= & 0.28 X_{1}+1.78 X_{2}+0.29 X_{3}+3.53 X_{1} X_{2} \\
& +0.02 X_{1} X_{3}+5.90 X_{2} X_{3}, \\
Y_{5}= & 0.03 X_{1}+0.56 X_{2}+0.06 X_{3}+0.89 X_{1} X_{2} \\
& -0.31 X_{1} X_{3}+1.55 X_{2} X_{3}, \\
Y_{6}= & 0.73 X_{1}+3.24 X_{2}+1.92 X_{3}+7.89 X_{1} X_{2} \\
& +2.42 X_{1} X_{3}+14.14 X_{2} X_{3} .
\end{aligned}
$$

\subsubsection{Decoction with Gamma Irradiation.}

$$
\begin{aligned}
Y_{1}= & 17.56 X_{1}+20.89 X_{2}+16.38 X_{3}+90.24 X_{1} X_{2} \\
& +67.88 X_{1} X_{3}+67.88 X_{2} X_{3}+114.00 X_{1} X_{2} X_{3}, \\
Y_{2}= & 0.32 X_{1}+0.30 X_{2}+0.99 X_{3}+1.75 X_{1} X_{2} \\
& +3.78 X_{1} X_{3}+4.49 X_{2} X_{3}, \\
Y_{3}= & 0.10 X_{1}+0.47 X_{2}-0.02 X_{3}+1.67 X_{1} X_{2} \\
& +0.48 X_{1} X_{3}+0.24 X_{2} X_{3},
\end{aligned}
$$




$$
\begin{aligned}
Y_{4}= & 0.48 X_{1}+1.89 X_{2}+0.01 X_{3}+6.17 X_{1} X_{2} \\
& +1.24 X_{1} X_{3}+1.28 X_{2} X_{3}, \\
Y_{5}= & 0.29 X_{1}+0.62 X_{2}+0.01 X_{3}+1.29 X_{1} X_{2} \\
& -0.34 X_{1} X_{3}+0.62 X_{2} X_{3}, \\
Y_{6}= & 1.19 X_{1}+3.27 X_{2}+1.00 X_{3}+10.88 X_{1} X_{2} \\
& +5.16 X_{1} X_{3}+6.62 X_{2} X_{3} .
\end{aligned}
$$

3.1.3. Infusion.

$$
\begin{aligned}
Y_{1}= & 11.29 X_{1}+17.55 X_{2}+11.98 X_{3}+99.33 X_{1} X_{2} \\
& +73.50 X_{1} X_{3}+58.07 X_{2} X_{3}, \\
Y_{2}= & 0.24 X_{1}+0.33 X_{2}+1.06 X_{3}+2.14 X_{1} X_{2} \\
& +4.31 X_{1} X_{3}+2.85 X_{2} X_{3} \\
Y_{3}= & 0.05 X_{1}+0.41 X_{2}-0.01 X_{3}+1.46 X_{1} X_{2} \\
& +0.46 X_{1} X_{3}+0.96 X_{2} X_{3} \\
Y_{4}= & 0.24 X_{1}+1.88 X_{2}+0.001 X_{3}+5.89 X_{1} X_{2} \\
& +1.79 X_{1} X_{3}+4.60 X_{2} X_{3}, \\
Y_{5}= & 0.02 X_{1}+0.71 X_{2}+0.05 X_{3}+1.49 X_{1} X_{2} \\
& +0.17 X_{1} X_{3}+1.15 X_{2} X_{3} \\
Y_{6}= & 0.55 X_{1}+3.33 X_{2}+1.11 X_{3}+10.98 X_{1} X_{2} \\
& +6.73 X_{1} X_{3}+9.56 X_{2} X_{3},
\end{aligned}
$$

Figure 3 shows the 3D response surfaces of model conditions of extraction yield, the individual content of phenolic compounds, and total content of four phenolic compounds. The high extraction yields of decoction groups with or without gamma irradiation and infusion group were found at the equal proportion of the three plants (Figure 3(a)). The extraction yields obtained from decoction and infusion were $16.33-49.33 \%$ and $11.83-45.50 \%$, respectively. The high gallic acid content of the decoction group without gamma irradiation was achieved at the low proportion of T. chebula with a medium proportion of T. bellirica and medium to the high proportion of $P$. emblica. The high gallic acid content of the decoction group with gamma irradiation and the infusion group was achieved at the low to medium proportion of T. chebula with the low to medium proportion of $T$. bellirica and the medium to high proportion of P. emblica (Figure $3(\mathrm{~b})$ ). The high corilagin content of the decoction group without gamma irradiation was achieved at the low proportion of T. chebula with the medium proportion of $T$. bellirica and the medium proportion of $P$. emblica. The high corilagin content of the decoction group with gamma irradiation and the infusion group was achieved at the low to medium proportion of $T$. chebula with the medium to high proportion of $T$. bellirica and the low proportion of $P$. emblica (Figure 3(c)). The high chebulagic acid content of the decoction group without gamma irradiation was achieved at the low to medium proportion of $T$. chebula with the medium to high proportion of T. bellirica and the low to medium proportion of $P$. emblica. The high chebulagic acid content of the decoction group with gamma irradiation was achieved at the low to medium proportion of $T$. chebula with the medium to high proportion of T. bellirica and the low proportion of $P$. emblica. The high chebulagic acid content of the infusion group was achieved at the low to medium proportion of T. chebula with the medium to high proportion of T. bellirica and the low to medium proportion of $P$. emblica (Figure 3(d)). The high chebulinic acid content of the decoction group without gamma irradiation was achieved at the low to medium proportion of $T$. chebula with the medium to high proportion of T. bellirica and the low to medium proportion of $P$. emblica. The high chebulinic acid content of the decoction group with gamma irradiation was achieved at the low to medium proportion of $T$. chebula with the medium to high proportion of T. bellirica and the low proportion of $P$. emblica. The high chebulinic acid content of the infusion group was achieved at the low to medium proportion of T. chebula with the medium to high proportion of $T$. bellirica and the low to medium proportion of $P$. emblica (Figure 3(e)). The high total content of four phenolic compounds of the decoction group without gamma irradiation was achieved at the low to medium proportion of $T$. chebula with the medium proportion of T. bellirica and the medium proportion of $P$. emblica. The high total content of four phenolic compounds of the decoction group with gamma irradiation and the infusion group was achieved at the low to medium proportion of $T$. chebula with the medium to high proportion of T. bellirica and the low to medium proportion of P. emblica (Figure 3(f)). In addition, the $3 \mathrm{D}$ response surfaces revealed that gamma irradiation slightly altered the content as well as pattern of the response surfaces of some phenolic compounds. Decomposition of some unstable substances or alteration of some phenolic compounds by gamma irradiation was previously reported [28-31]. Furthermore, extraction techniques gave different phenolic compounds' content. The decoction seems to provide the higher extraction yield and content of phenolic compounds compared with the infusion. The author mentioned that infusion might be an alternative method of Triphala because it seems to be an energy-saving manner compared to decoction.

3.2. Interaction of Herbal Ingredients Contained in the Triphala Recipe. The mathematic equations used to predict each response $\left(Y_{7}\right.$ to $\left.Y_{12}\right)$ are shown in Equations (20)(37). Among the proportion of T. chebula $\left(X_{1}\right)$, T. bellirica $\left(X_{2}\right)$, and P. emblica $\left(X_{3}\right)$, all $X_{1}, X_{2}$, and $X_{3}$ seem to have an equal effect on $\mathrm{CI}$ of the extraction yield $\left(Y_{7}\right)$, gallic acid content $\left(Y_{8}\right)$, corilagin content $\left(Y_{9}\right)$, chebulagic acid $\left(Y_{10}\right)$, chebulinic acid $\left(Y_{11}\right)$, and total content of the four phenolic compounds $\left(Y_{12}\right)$ of all groups. However, the interaction of the three plants significantly affected all dependent variables.

\subsubsection{Decoction without Gamma Irradiation}

$$
\begin{aligned}
Y_{7}= & 0.99 X_{1}+0.99 X_{2}+0.98 X_{3}-2.05 X_{1} X_{2} \\
& -1.90 X_{1} X_{3}-1.81 X_{2} X_{3},
\end{aligned}
$$



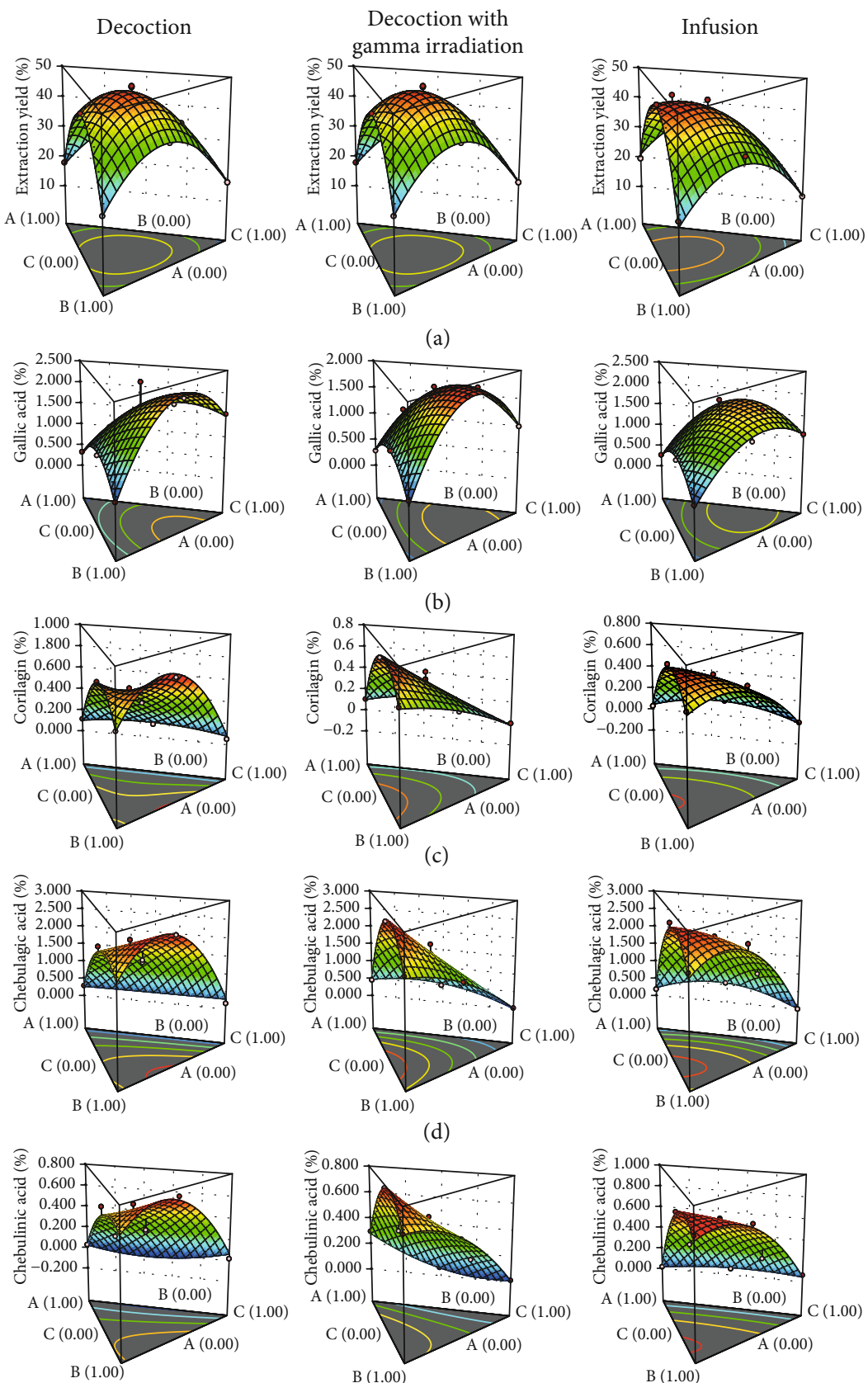

(d)
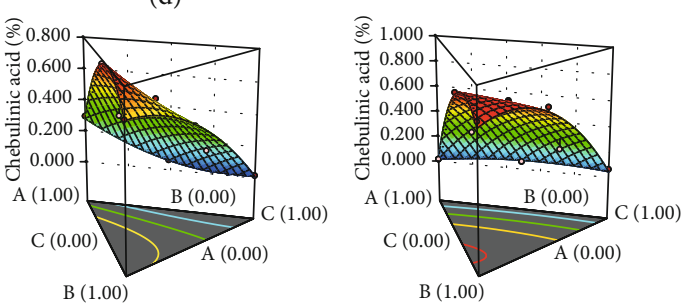

(e)
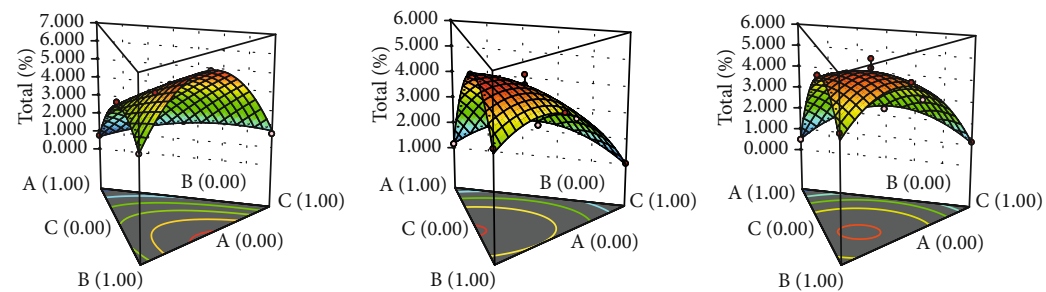

(f)

Figure 3: 3D response surfaces of model conditions of (a) extraction yield, (b) gallic acid content, (c) corilagin content, (d) chebulagic acid content, (e) chebulinic acid content, and (f) total content of four phenolic compounds. $A, B$, and $C$ were T. chebula, T. bellirica, and P. emblica, respectively. 


$$
\begin{aligned}
Y_{8}= & 0.98 X_{1}+0.99 X_{2}+1.01 X_{3}-2.10 X_{1} X_{2} \\
& -1.45 X_{1} X_{3}-2.03 X_{2} X_{3}, \\
Y_{9}= & 0.98 X_{1}+1.00 X_{2}+0.93 X_{3}-1.88 X_{1} X_{2} \\
& -1.21 X_{1} X_{3}-2.36 X_{2} X_{3}, \\
Y_{10}= & 0.98 X_{1}+1.01 X_{2}+0.93 X_{3}-1.82 X_{1} X_{2} \\
& -1.31 X_{1} X_{3}-2.38 X_{2} X_{3}, \\
Y_{11}= & 0.98 X_{1}+1.02 X_{2}+0.91 X_{3}-1.89 X_{1} X_{2} \\
& -0.57 X_{1} X_{3}-2.42 X_{2} X_{3}, \\
Y_{12}= & 0.98 X_{1}+1.00 X_{2}+0.96 X_{3}-1.92 X_{1} X_{2} \\
& -1.37 X_{1} X_{3}-2.24 X_{2} X_{3} .
\end{aligned}
$$

3.2.2. Decoction with Gamma Irradiation.

$$
\begin{aligned}
Y_{7}= & 0.99 X_{1}+0.99 X_{2}+0.98 X_{3}-2.05 X_{1} X_{2} \\
& -1.90 X_{1} X_{3}-1.81 X_{2} X_{3}, \\
Y_{8}= & 0.96 X_{1}+1.00 X_{2}+0.99 X_{3}-2.01 X_{1} X_{2} \\
& -2.15 X_{1} X_{3}-2.20 X_{2} X_{3}, \\
Y_{9}= & 0.99 X_{1}+1.01 X_{2}+1.00 X_{3}-2.28 X_{1} X_{2} \\
& -2.56 X_{1} X_{3}-0.51 X_{2} X_{3}, \\
Y_{10}= & 0.97 X_{1}+1.02 X_{2}+0.97 X_{3}-2.17 X_{1} X_{2} \\
& -2.06 X_{1} X_{3}-0.87 X_{2} X_{3}, \\
Y_{11}= & 0.99 X_{1}+0.99 X_{2}+0.99 X_{3}-1.68 X_{1} X_{2} \\
& +5.85 X_{1} X_{3}-1.29 X_{2} X_{3}-35.69 X_{1}^{2} X_{2} X_{3} \\
& +18.20 X_{1} X_{2}^{2} X_{3}-41.06 X_{1} X_{2} X_{3}^{2}, \\
Y_{12}= & 1.00 X_{1}+1.00 X_{2}+1.00 X_{3}-2.15 X_{1} X_{2} \\
& -2.04 X_{1} X_{3}-1.68 X_{2} X_{3}-6.19 X_{1}^{2} X_{2} X_{3} \\
& +13.91 X_{1} X_{2}^{2} X_{3}-2.67 X_{1} X_{2} X_{3}^{2} .
\end{aligned}
$$

\subsubsection{Infusion.}

$$
\begin{aligned}
Y_{7}= & 1.00 X_{1}+1.00 X_{2}+1.00 X_{3}-2.29 X_{1} X_{2} \\
& -2.16 X_{1} X_{3}-2.05 X_{2} X_{3}-3.66 X_{1}^{2} X_{2} X_{3} \\
& +14.24 X_{1} X_{2}^{2} X_{3}-1.44 X_{1} X_{2} X_{3}^{2}, \\
Y_{8}= & 0.97 X_{1}+1.00 X_{2}+1.00 X_{3}-2.14 X_{1} X_{2} \\
& -2.26 X_{1} X_{3}-1.65 X_{2} X_{3}, \\
Y_{9}= & 1.00 X_{1}+1.00 X_{2}+1.00 X_{3}-2.48 X_{1} X_{2} \\
& -3.54 X_{1} X_{3}-2.22 X_{2} X_{3}-6.96 X_{1}^{2} X_{2} X_{3} \\
& +25.33 X_{1} X_{2}^{2} X_{3}+0.68 X_{1} X_{2} X_{3}^{2}, \\
Y_{10}= & 1.00 X_{1}+1.00 X_{2}+1.00 X_{3}-2.35 X_{1} X_{2} \\
& -3.34 X_{1} X_{3}-2.23 X_{2} X_{3}-5.36 X_{1}^{2} X_{2} X_{3} \\
& +26.86 X_{1} X_{2}^{2} X_{3}-3.68 X_{1} X_{2} X_{3}^{2},
\end{aligned}
$$

$$
\begin{aligned}
Y_{11}= & 1.01 X_{1}+1.01 X_{2}+1.01 X_{3}-1.92 X_{1} X_{2} \\
& -1.40 X_{1} X_{3}-1.63 X_{2} X_{3}-21.43 X_{1}^{2} X_{2} X_{3} \\
& +22.80 X_{1} X_{2}^{2} X_{3}-0.58 X_{1} X_{2} X_{3}^{2} \\
Y_{12}= & 1.00 X_{1}+1.00 X_{2}+1.00 X_{3}-2.28 X_{1} X_{2} \\
& -2.61 X_{1} X_{3}-2.02 X_{2} X_{3}-9.78 X_{1}^{2} X_{2} X_{3} \\
& +20.65 X_{1} X_{2}^{2} X_{3}-1.11 X_{1} X_{2} X_{3}^{2} .
\end{aligned}
$$

Figure 4 shows the contour plots of model conditions of CI of extraction yield, the individual content of phenolic compounds, and total content of four phenolic compounds. It was focused on the low CI value which reflects a high level of interaction. The low CI of the extraction yield of decoction groups with or without gamma irradiation was found at the equal proportion of the three plants. In case of the infusion group, the low extraction yield was found at the medium proportion of T. chebula with the low to medium proportion of $T$. bellirica and the medium proportion of $P$. emblica (Figure 4(a)). The low CI of gallic acid content of decoction groups with or without gamma irradiation and the infusion group were achieved at the equal proportion of the three plants (Figure 4(b)). The low CI of corilagin and chebulagic acid content of the decoction group without gamma irradiation was achieved at the low to medium proportion of $T$. chebula with the medium proportion of T. bellirica and the medium proportion of $P$. emblica. The low CI of corilagin and chebulagic acid content of the decoction group with gamma irradiation was achieved at the equal proportion of the three plants. The low CI of corilagin and chebulagic acid content of the infusion group was achieved at the medium proportion of T. chebula with the low proportion of T. bellirica and the medium proportion of P. emblica (Figures 4(c) and 4(d)). The low CI of chebulinic acid content of the decoction group without gamma irradiation was achieved at the low proportion of $T$. chebula with the medium proportion of $T$. bellirica and the medium proportion of $P$. emblica. The low CI of chebulinic acid content of the decoction group with gamma irradiation was achieved at the low to high proportion of T. chebula with the medium to high proportion of $T$. bellirica and the low to high proportion of $P$. emblica. The low CI of chebulinic acid content of the infusion group was achieved at the medium proportion of $T$. chebula with the low to medium proportion of $T$. bellirica and the medium proportion of $P$. emblica (Figure 4(e)). The low CI of total content of four phenolic compounds of the decoction group without gamma irradiation was achieved at the equal proportion of the three plants. The low CI of total content of four phenolic compounds of the decoction group with gamma irradiation and the infusion group was achieved at the medium proportion of $T$. chebula with the low to medium proportion of T. bellirica and the medium proportion of $P$. emblica (Figure 4(f)).

The correlation plots between predicted vs. actual values of model conditions of CI of extraction yield, gallic acid content, corilagin content, chebulagic acid content, chebulinic acid content, and total content of four phenolic compounds are shown in Figure 5. Relative high $R^{2}$ values indicated that the prediction of the Design-Expert ${ }^{\circledR}$ software was precise and 

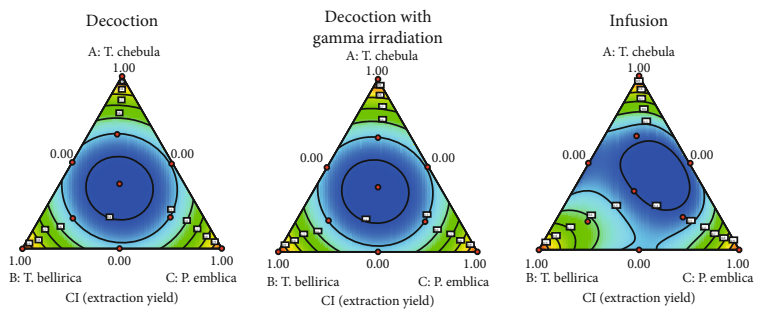

(a)
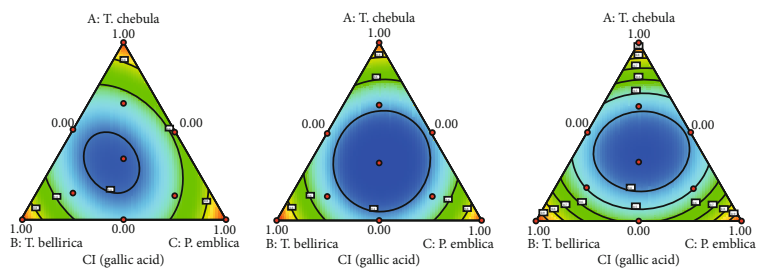

(b)
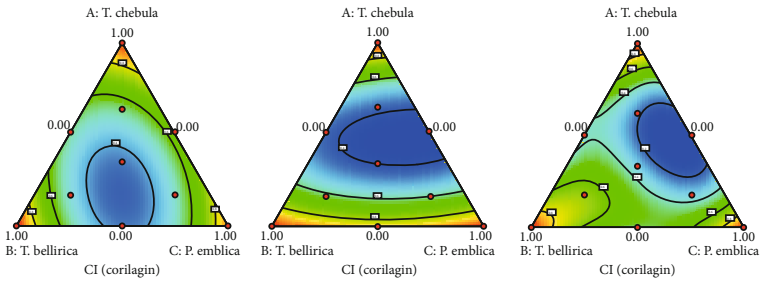

(c)
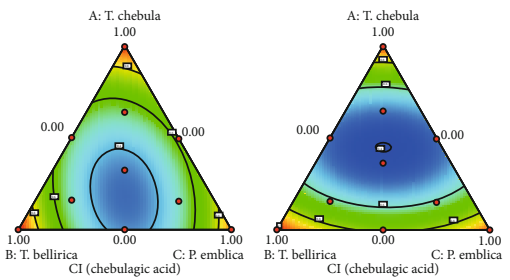

(d)
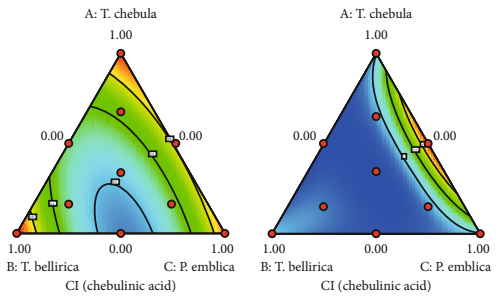

(e)
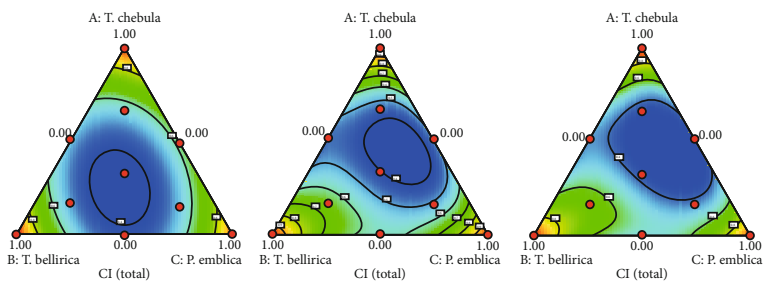

(f)

Figure 4: Contour plots of model conditions of combination index of (a) extraction yield, (b) gallic acid content, (c) corilagin content, (d) chebulagic acid content, (e) chebulinic acid content, and (f) total content of four phenolic compounds. reliable. The correlation plots between internally studentized residuals vs. run numbers of model conditions of $\mathrm{CI}$ of extraction yield, gallic acid content, corilagin content, chebulagic acid, chebulinic acid, and total content of four phenolic compounds are shown in Figure 6. All data were distributed within the limit (red line). They indicated that the data distribution was within $95 \%$ confident interval. It could be confirmed that the prediction by Design-Expert ${ }^{\circledR}$ software was stable [32-36].

Figure 7(a) shows that contour plots of the desirability of the optimal condition provided the simultaneous minimizing $\mathrm{CI}$ of the extraction yield and total content of the four phenolic compounds. The maximized synergistic effect was found at the proportion of T. chebula, T. bellirica, and P. emblica of $0.33: 0.33: 0.33,0.35: 0.30: 0.35$, and $0.43: 0.27: 0.30$ for the decoction without gamma irradiation group, the decoction with gamma irradiation group, and the infusion group, respectively. Figures 7(b) and 7(c) show the overlay plots of CI of extraction yield and total content of four phenolic compounds for which the values were less than 0.5 and 0.4, respectively. The yellow area of the overlay plots indicated the proportion of T. chebula, T. bellirica, and P. emblica providing the $\mathrm{CI}$ values of extraction yield and total content of four phenolic compounds of less than 0.5 (Figure 7(b)) or less than 0.4 (Figure $7(\mathrm{c})$ ). According to the equal proportion of the three plants, the traditional Triphala recipe, the CI values were less than 0.5 . It was indicated that the combination of equal proportion of T. chebula, T. bellirica, and $P$. emblica could promote the combination effect greater than the expected additive effect given by the summation of the individual effect for at least $200 \%$. Because of the broad yellow area of overlay plots, the synergism could occur in a wide range of plant proportions apart from the equal ratio. When focused on the CI values of less than 0.4 , the traditional Triphala recipe still promoted the combination effect greater than the expected additive effect for at least $250 \%$. It could be established that the traditional Triphala recipe of decoction without gamma irradiation and infusion groups exhibited the positive interaction of more than 2.5 times of the individual effect of the single plant while the decoction group with gamma irradiation exhibited the positive interaction of 2 to 2.5 times of the individual effect of the single plant.

The interrelationship of the herbs in the herbal recipe could occur in different ways: two or more herbs reinforcing each other called synergism, herbs strengthening the effect of another herb called assisting, herbs reducing the curative effect of another herb called antagonism, and herbs increasing toxicity of another herb called rejection [37]. The synergism occurred among herbs or other components in the prescription, among effective parts of herbs, or among bioactive compounds of herbs [25]. In the case of the Triphala recipe, the positive interaction could be promoted by the herbal ingredients contained in the recipe or among active compounds of the recipe, which was investigated using validated HPLC combined 3D response surface analysis. The authors suggested that the positive interaction could be used as a tool to describe the mechanism of the synergism of the herbal formula. This data scientifically proved that the traditional Triphala recipe was appropriate to use due to their positive interaction based on chemical analysis point of view. 

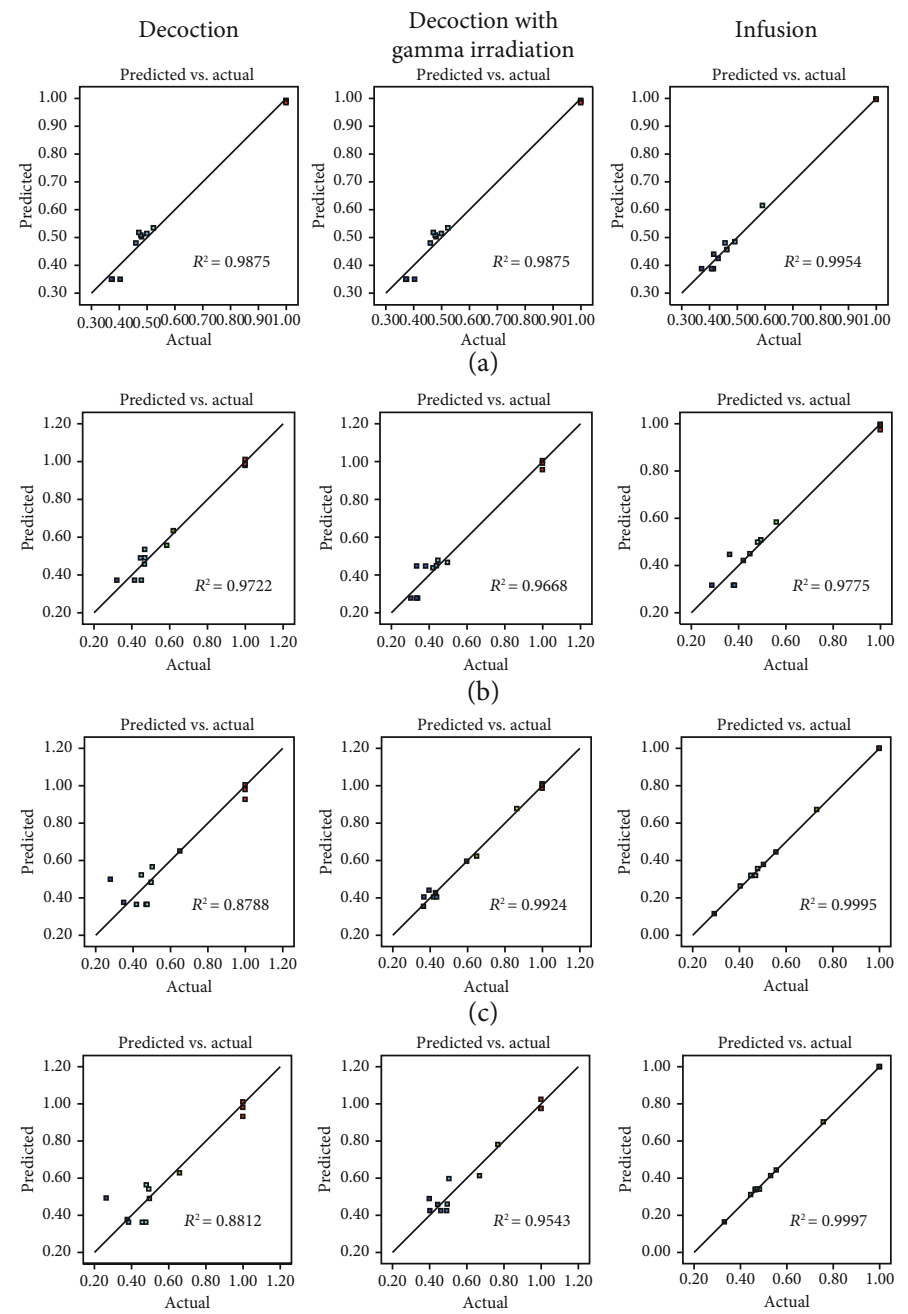

(c)
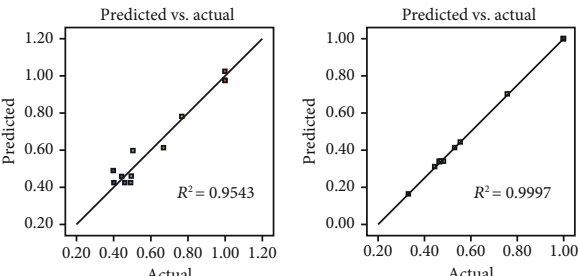

Actual
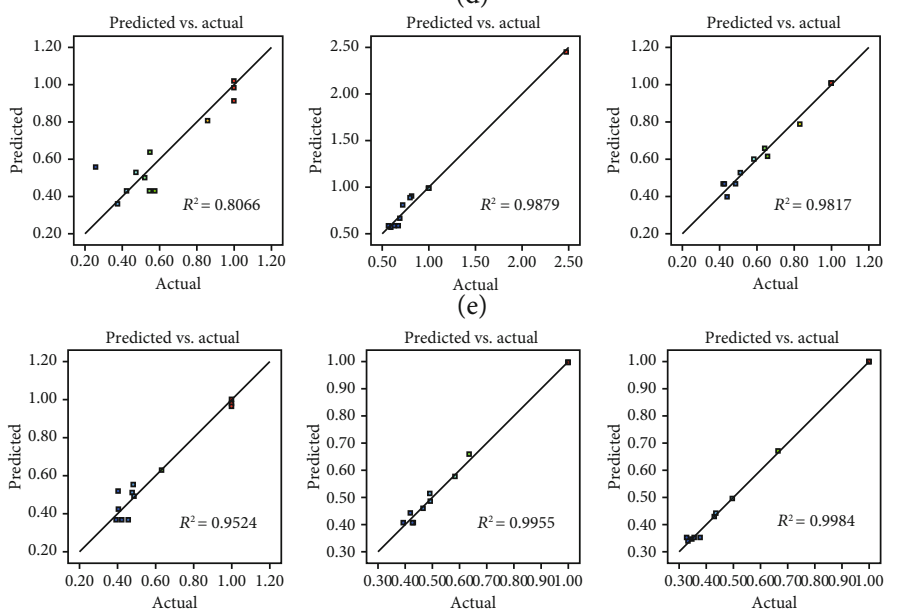

(f)

Figure 5: Predicted vs. actual value plots of model conditions of (a) extraction yield, (b) gallic acid content, (c) corilagin content, (d) chebulagic acid content, (e) chebulinic acid content, and (f) total content of four phenolic compounds.

\section{Conclusions}

The investigation of the interaction of the three herbal ingredients contained in the Triphala recipe was designed based on the simplex lattice experimental design. The four phenolic compounds were selected as a chemical marker for determination of the interaction based on chemical analysis point of view. Results showed that the extraction techniques affected the extraction yield as well as content of phenolic compounds. The decoction gave more extraction yield and 

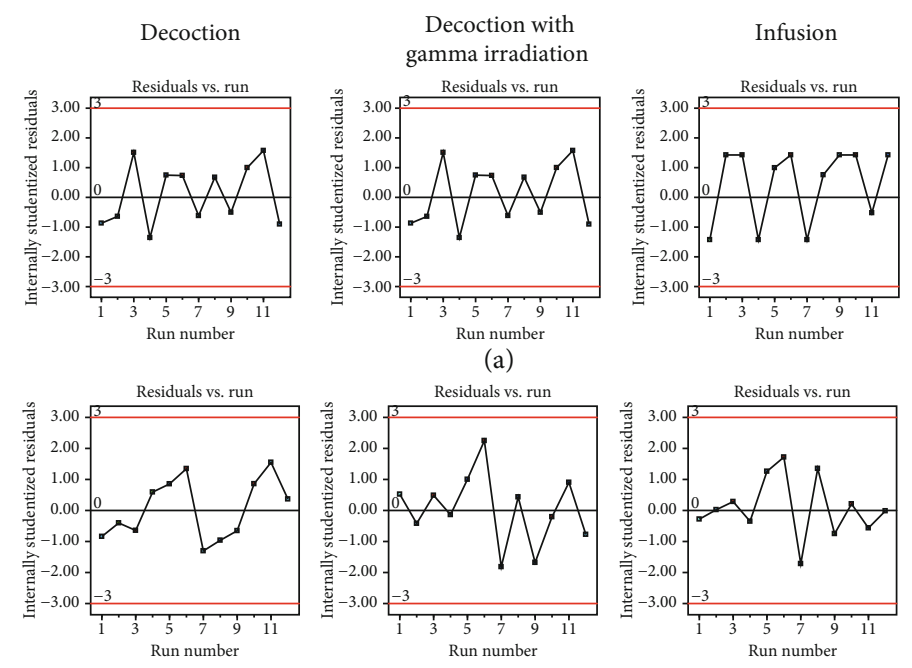

(a)
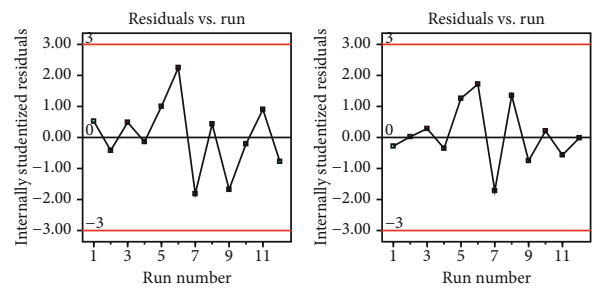

(b)
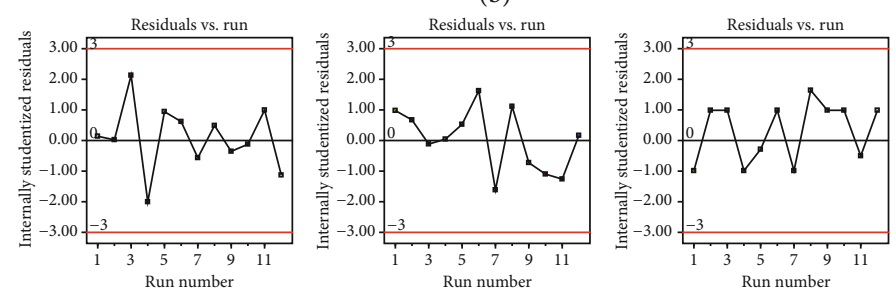

(c)
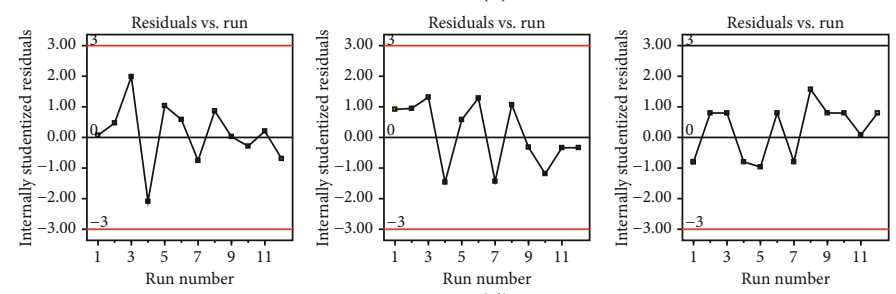

(d)
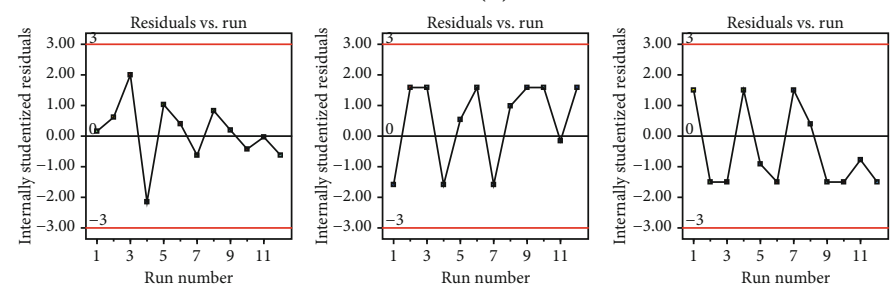

(e)
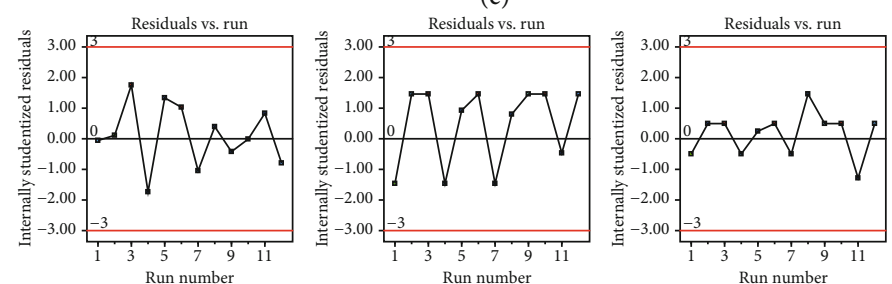

(f)

Figure 6: Residuals vs. run plots of model conditions of (a) extraction yield, (b) gallic acid content, (c) corilagin content, (d) chebulagic acid content, (e) chebulinic acid content, and (f) total content of four phenolic compounds.

phenolic compound content compared with infusion. In addition, gamma irradiation could slightly alter the content of phenolic compounds. According to the interaction of extraction yield and total content of phenolic compounds, the positive interaction seems to be found at the equal proportion of T. chebula, T. bellirica, and P. emblica, which was similar to the traditional use. It was found that at those ratios, the extraction yield and total content of phenolic compounds increased at least two times the individual effect of each plant. In summary, the data of this work supported that the equal proportion of T. chebula, T. bellirica, and P. emblica in the Triphala recipe was already appropriate. 

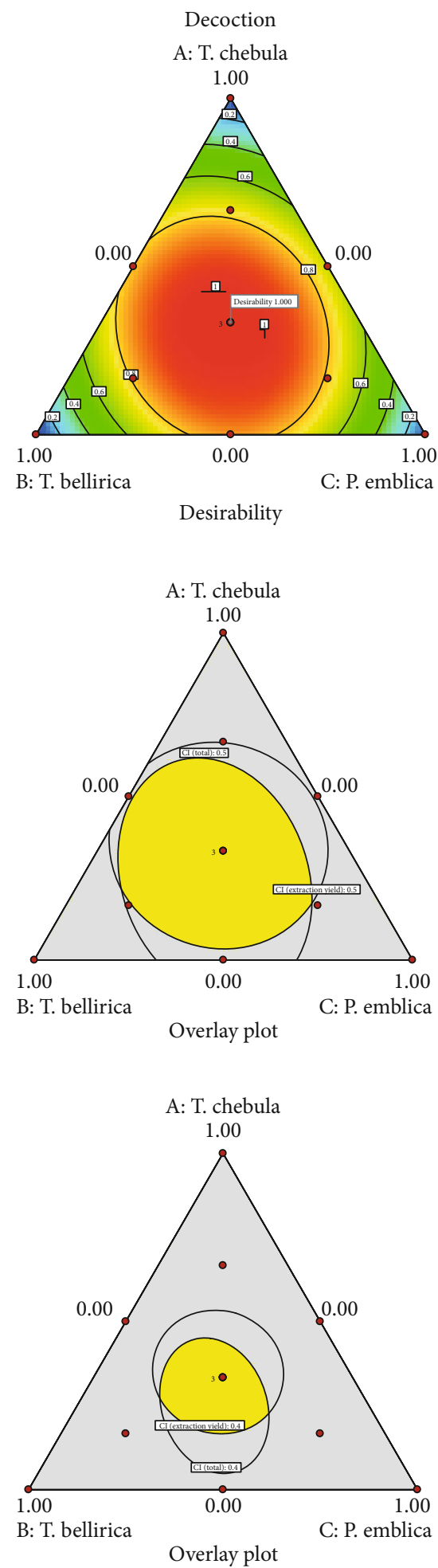

Decoction with gamma irradiation

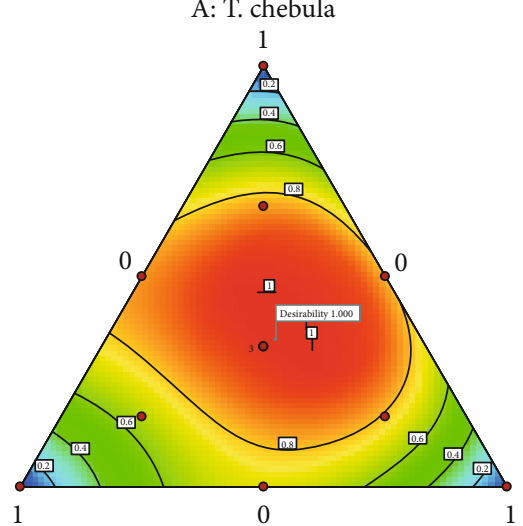

B: T. bellirica

Desirability

(a)

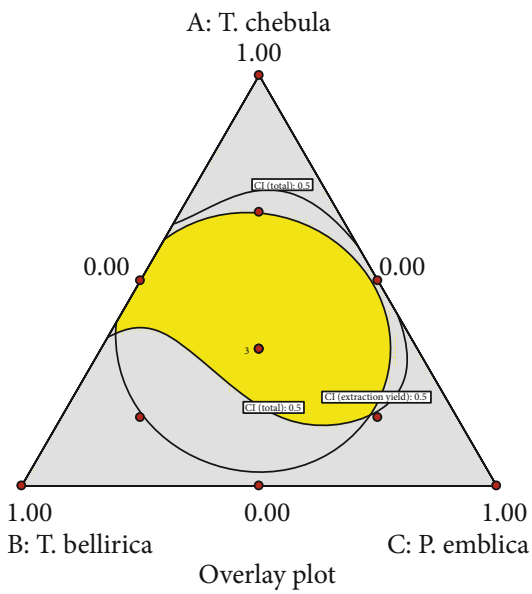

(b)

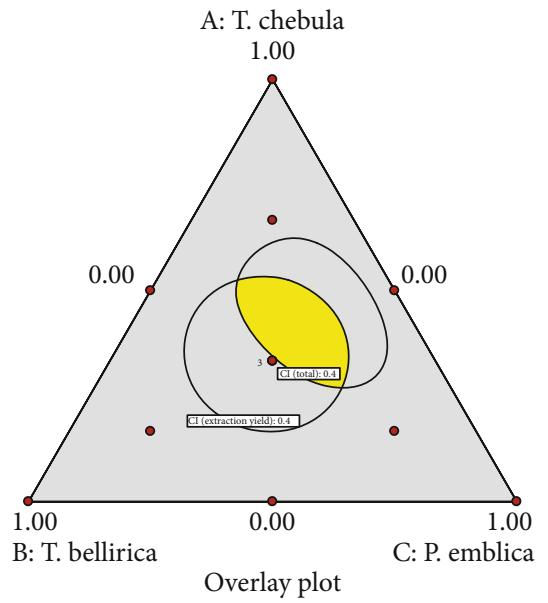

(c)
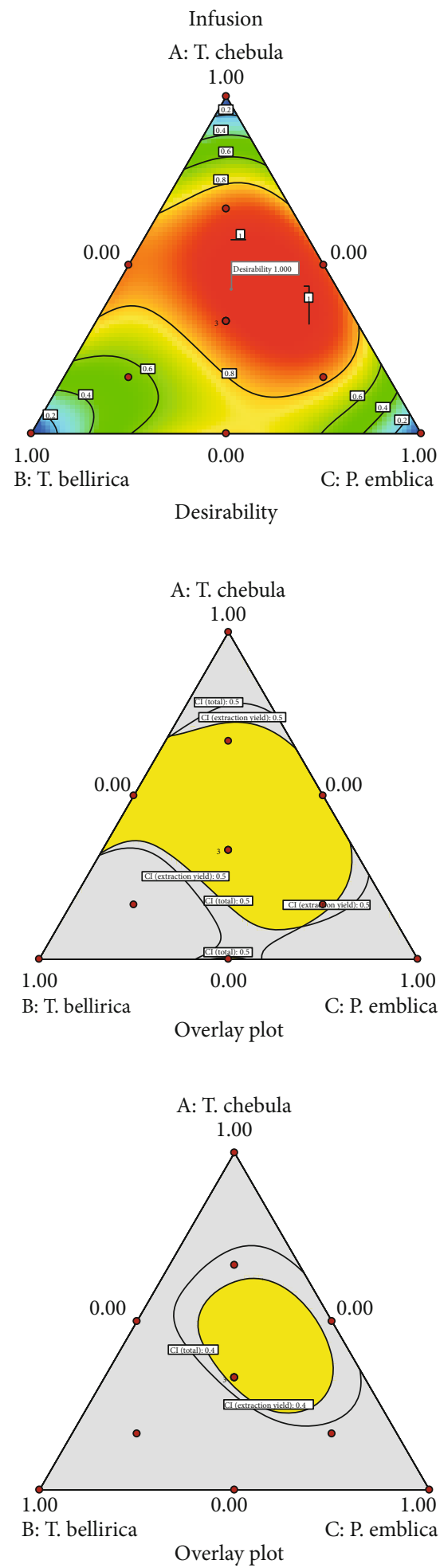

Overlay plot

Figure 7: Desirability plots (a) of the optimal condition provided the simultaneous minimizing combination index of the extraction yield and total content of the four phenolic compounds and overlay plots (b, c) of the combination index of the extraction yield and total content of four phenolic compounds which the combination indices of both factors were lower than (b) 0.5 and (c) 0.4 . 


\section{Data Availability}

The data used to support the findings of this study are available from the corresponding author upon request.

\section{Conflicts of Interest}

All authors declare that they have no conflicts of interest.

\section{Acknowledgments}

We would like to acknowledge Mr. Wipas Wiboolsamai and Miss Patcharawadee Cheerawattana for research assistance. We also acknowledge Prof. Dr. J. E. Moreton, School of Pharmacy, University of Maryland, at Baltimore, USA, for reading and editing English language in this paper. This research did not receive any specific grant from funding agencies in the public, commercial, or not-for-profit sectors.

\section{References}

[1] C. T. Peterson, K. Denniston, and D. Chopra, "Therapeutic uses of Triphala in Ayurvedic medicine," Journal of Alternative and Complementary Medicine, vol. 23, no. 8, pp. 607-614, 2017.

[2] A. Tarasiuk, P. Mosińska, and J. Fichna, "Triphala: current applications and new perspectives on the treatment of functional gastrointestinal disorders," Chinese Medicine, vol. 13, no. 1, p. 39, 2018.

[3] V. Pawar, P. Lahorkar, and D. B. Anantha Narayana, "Development of a RP-HPLC method for analysis of Triphala Curna and its applicability to test variations in Triphala Curna preparations," Indian Journal of Pharmaceutical Sciences, vol. 71, no. 4, pp. 382-386, 2009.

[4] L. Charoenchai, P. Pathompak, F. Madaka, S. Settharaksa, and W. Saingam, "HPLC-MS profiles and quantitative analysis of Triphala formulation," Bulletin of Health, Science and Technology, vol. 14, no. 1, pp. 57-67, 2016.

[5] S. R. Varma, T. O. Sivaprakasam, A. Mishra et al., "Protective effects of Triphala on dermal fibroblasts and human keratinocytes," PLoS One, vol. 11, no. 1, article e0145921, 2016.

[6] E. Vamanu and S. Nita, "Antioxidant capacity and the correlation with major phenolic compounds, anthocyanin, and tocopherol content in various extracts from the wild edible Boletus edulis mushroom," BioMed Research International, vol. 2013, Article ID 313905, 11 pages, 2013.

[7] E. A. Turumtay, F. İslamoğlu, D. Çavuş, H. Şahin, H. Turumtay, and B. Vanholme, "Correlation between phenolic compounds and antioxidant activity of Anzer tea (Thymus praecox Opiz subsp. caucasicus var. caucasicus)," Industrial Crops and Products, vol. 52, pp. 687-694, 2014.

[8] G. Piluzza and S. Bullitta, "Correlations between phenolic content and antioxidant properties in twenty-four plant species of traditional ethnoveterinary use in the Mediterranean area," Pharmaceutical Biology, vol. 49, no. 3, pp. 240-247, 2010.

[9] X. Li, X. Wu, and L. Huang, "Correlation between antioxidant activities and phenolic contents of radix Angelicae sinensis (Danggui)," Molecules, vol. 14, no. 12, pp. 5349-5361, 2009.

[10] M. H. Carlsen, B. L. Halvorsen, K. Holte et al., "The total antioxidant content of more than 3100 foods, beverages, spices, herbs and supplements used worldwide," Nutrition Journal, vol. 9, no. 1, p. 3, 2010.

[11] S. N. Save and S. Choudhary, "Effects of triphala and guggul aqueous extracts on inhibition of protein fibrillation and dissolution of preformed fibrils," RSC Advances, vol. 7, no. 33, pp. 20460-20468, 2017.

[12] X. Zhou, V. Razmovski-Naumovski, A. Kam et al., "Synergistic study of a Danshen (Salvia Miltiorrhizae Radix et Rhizoma) and Sanqi (Notoginseng Radix et Rhizoma) combination on cell survival in EA.hy926 cells," BMC Complementary and Alternative Medicine, vol. 19, no. 1, p. 50, 2019.

[13] M. Bahmani, M. Taherikalani, M. Khaksarian et al., "The synergistic effect of hydroalcoholic extracts ofOriganum vulgare,Hypericum perforatumand their active components carvacrol and hypericin againstStaphylococcus aureus," Future Science $O A$, vol. 5, no. 3, article FSO371, 2019.

[14] I. Y. Bae, B.-Y. Kwak, and H. G. Lee, "Synergistic antiradical action of natural antioxidants and herbal mixture for preventing dioxin toxicity," Food Science and Biotechnology, vol. 21, no. 2, pp. 491-496, 2012.

[15] A. Bag and R. R. Chattopadhyay, "Evaluation of synergistic antibacterial and antioxidant efficacy of essential oils of spices and herbs in combination," PLoS One, vol. 10, no. 7, article e0131321, 2015.

[16] K. M. Lau, K. K. Lai, C. L. Liu et al., "Synergistic interaction between Astragali Radix and Rehmanniae Radix in a Chinese herbal formula to promote diabetic wound healing," Journal of Ethnopharmacology, vol. 141, no. 1, pp. 250-256, 2012.

[17] F. S. Youssef, R. M. Labib, O. A. Eldahshan, and A. N. B. Singab, "Synergistic hepatoprotective and antioxidant effect of artichoke, fig, blackberry herbal mixture on HepG2 cells and their metabolic profiling using NMR coupled with chemometrics," Chemistry \& Biodiversity, vol. 14, no. 12, article e1700206, 2017.

[18] M. Nikkhah, M. Hashemi, M. B. Habibi Najafi, and R. Farhoosh, "Synergistic effects of some essential oils against fungal spoilage on pear fruit," International Journal of Food Microbiology, vol. 257, pp. 285-294, 2017.

[19] Y. A. Crespo, L. R. Bravo Sánchez, Y. G. Quintana, A. S. T. Cabrera, A. Bermúdez del Sol, and D. M. G. Mayancha, "Evaluation of the synergistic effects of antioxidant activity on mixtures of the essential oil from Apium graveolens L., Thymus vulgaris L. and Coriandrum sativum L. using simplex-lattice design," Heliyon, vol. 5, no. 6, article e01942, 2019.

[20] A. Rosato, A. Carocci, A. Catalano et al., "Elucidation of the synergistic action of Mentha piperita essential oil with common antimicrobials," PLoS One, vol. 13, no. 8, article e0200902, 2018.

[21] C. Alaoui Jamali, A. Kasrati, M. Fadli, L. Hassani, D. Leach, and A. Abbad, "Synergistic effects of three Moroccan thyme essential oils with antibiotic cefixime," Phytothérapie, vol. 16, no. S1, pp. S149-S154, 2018.

[22] Y. El Atki, I. Aouam, F. El Kamari et al., “Antibacterial activity of cinnamon essential oils and their synergistic potential with antibiotics," Journal of Advanced Pharmaceutical Technology \& Research, vol. 10, no. 2, pp. 63-67, 2019.

[23] S. Li and B. Zhang, "Traditional Chinese medicine network pharmacology: theory, methodology and application," Chinese Journal of Natural Medicines, vol. 11, no. 2, pp. 110-120, 2013.

[24] X. Zhou, S. W. Seto, D. Chang et al., "Synergistic effects of Chinese herbal medicine: a comprehensive review of methodology 
and current research," Frontiers in Pharmacology, vol. 7, p. 201, 2016.

[25] H. Yuan, Q. Ma, H. Cui et al., "How can synergism of traditional medicines benefit from network pharmacology?," Molecules, vol. 22, no. 7, article 1135, 2017.

[26] A. Manoraj, V. Thevanesam, B. M. R. Bandara, A. Ekanayake, and V. Liyanapathirana, "Synergistic activity between Triphala and selected antibiotics against drug resistant clinical isolates," BMC Complementary and Alternative Medicine, vol. 19, no. 1, p. 199, 2019.

[27] J. Foucquier and M. Guedj, "Analysis of drug combinations: current methodological landscape," Pharmacology Research \& Perspectives, vol. 3, no. 3, article e00149, 2015.

[28] A. G. Chmielewski and W. Migdal, "Radiation decontamination of herbs and spices," Nukleonika, vol. 50, no. 4, pp. 179184, 2005.

[29] E. Pereira, L. Barros, A. Antonio et al., "Is gamma radiation suitable to preserve phenolic compounds and to decontaminate mycotoxins in aromatic plants? A case-study with Aloysia citrodora Paláu," Molecules, vol. 22, no. 3, p. 347, 2017.

[30] T. Gumus, S. Albayrak, O. Sagdic, and M. Arici, "Effect of gamma irradiation on total phenolic contents and antioxidant activities of Satureja Hortensis, Thymus Vulgaris, and Thymbra Spicata from Turkey," International Journal of Food Properties, vol. 14, no. 4, pp. 830-839, 2011.

[31] V. C. Ito, A. Alberti, S. Avila, M. Spoto, A. Nogueira, and G. Wosiacki, "Effects of gamma radiation on the phenolic compounds and in vitro antioxidant activity of apple pomace flour during storage using multivariate statistical techniques," Innovative Food Science and Emerging Technologies, vol. 33, pp. 251-259, 2016.

[32] S. Duangjit, L. M. Mehr, M. Kumpugdee-Vollrath, and T. Ngawhirunpat, "Role of simplex lattice statistical design in the formulation and optimization of microemulsions for transdermal delivery," Biological \& Pharmaceutical Bulletin, vol. 37, no. 12, pp. 1948-1957, 2014.

[33] S. Duangjit, Y. Obata, H. Sano et al., "Menthosomes, novel ultradeformable vesicles for transdermal drug delivery: optimization and characterization," Biological \& Pharmaceutical Bulletin, vol. 35, no. 10, pp. 1720-1728, 2012.

[34] C. Monton and C. Luprasong, "Effect of temperature and duration time of maceration on nitrate content of Vernonia cinerea (L.) Less.: Circumscribed central composite design and method validation," International Journal of Food Science, vol. 2019, Article ID 1281635, 8 pages, 2019.

[35] C. Monton, C. Luprasong, and L. Charoenchai, "Convection combined microwave drying affect quality of volatile oil compositions and quantity of curcuminoids of turmeric raw material," Revista Brasileira de Farmacognosia, vol. 29, no. 4, pp. 434-440, 2019.

[36] C. Monton, C. Luprasong, and L. Charoenchai, "Acceleration of turmeric drying using convection and microwave-assisted drying technique: an optimization approach," Journal of Food Processing and Preservation, vol. 43, no. 9, article e14096, 2019.

[37] W. Jia, W. Y. Gao, Y. Q. Yan et al., "The rediscovery of ancient Chinese herbal formulas," Phytotherapy Research, vol. 18, no. 8, pp. 681-686, 2004. 